\title{
PRICING FORWARD-FUTURES SPREAD BASED ON COPULAS WITH STOCHASTIC SIMULATION
}

\author{
YUQI PU ${ }^{\mathrm{a}}$ AND SEKI KIM ${ }^{\mathrm{b}, *}$
}

\begin{abstract}
This paper focuses on computational contractual distinctions as an explanation for the spread between a forward contract and a similar futures contract which is derived and investigated. We evaluate this spread by constructing a time series model, which was established based on copula functions, and also show that the forward-futures spread is more significant for long maturity.
\end{abstract}

\section{INTRODUCTION}

Forward and futures contracts are two seemingly similar financial instruments. Both are obligations to buy or sell a certain asset at an agreed price at a specified date in the future. Yet, the two instruments differ [6]. Whereas the forward is only settled at maturity, the futures contract is settled continuously through its lifespan. In other words, with the forward contract, cash is exchanged only at maturity, whereas with the futures contract, it is exchanged daily between contract initiation and maturity. This difference in cash flows has an impact on the value of the contracts.

It is our goal to show under which conditions the values of a forward and a futures contract are equal, and, if they differ, but how much. The difference in value between a forward contract and a similar futures contract is usually called forward-futures spread. Cox, Ingersoll, and Ross [2] analyze the relationship between forward and futures prices in a framework with stochastic interest rate. Grinblatt and Jegadeesh [5] compare the futures price with the forward price implied by the term structure of

\footnotetext{
Received by the editors January 14, 2014. Revised February 16, 2014. Accepted Feb. 17, 2014 2010 Mathematics Subject Classification. Primary 91G30, 65C20; Secondary 91G60 .

Key words and phrases. copulas, forward-futures spread, time series model, stochastic simulation. This work was supported by Basic Science Research Program through the National Research Foundation of Korea(NRF) funded by the Ministry of Education, Science and Technology(20100010694).

${ }^{*}$ Corresponding author.
} 
spot London Interbank Offered rates. Murawski [8] develop intuitive expressions for the forward-futures spread taking into account the probability of counterparty default. In this work, the methodology which is suited in pricing this spread is provided by generating stochastic processes with a given structure prescribed in terms of copula. The analysis of copula functions is started from the approach suggested by Nelsen [9]. Roughly speaking, the copula is a function that joins the marginal distributions of random variables in a multivariate distribution describing the joint behavior. Or statistically speaking, the copula connects a multivariate distribution to its marginals in such a way that it captures the entire dependence structure in the multivariate distribution. The importance of copulas in modeling the distribution of a multivariate random variable is justified by the Sklar's theorem: any multivariate distribution can be expressed as its copula function evaluated at its marginal distribution function; and any copula function when evaluated at any marginal distributions is a multivariate distribution. Hence the information in the joint distribution is decomposed into those in the marginal distributions and that in the copula function. Consequently copulas allow one to model the marginal distributions and the dependence structure of a multivariate random variable separately. Moreover, the copula measure of dependence is invariant to any increasing transformation of individual series.

In the copula approach to time series modeling, the finite dimensional distributions of the time series are generated by copulas. Chen and Fan [1] and Pumi and Lopes [10] exhibit the simulation of time series based on some prescribed copula. By coupling different marginal distributions with different copula functions, copula-based time series models are able to model the dependence structure and the marginal behavior of a time series separately, allowing for a wide variety of marginal behaviors (such as skewness and fat tailed-ness) and dependence properties (such as asymmetric dependence and positive tail dependence). This separate modeling of the temporal dependence and the marginal behavior is particularly important when it is known that the dependence structure and the marginal properties of a time series are affected by different exogenous variables, which can easily modeled via the parametric copula approach.

This paper is organized as follows. Section 2 provides an overview of copula functions, including the measure of concordance. In Section 3, the forward contract and the similar futures contract as well as the difference between them are investigated. Section 4 establishes the copula-based time series model and Section 5 gives the 
evaluation of forward-futures spread based on this model. The conclusion of this paper is in Section 6.

\section{Copula Functions}

In this section we give the precise definition and important fundamental properties as well as some examples that are necessary for this work. We then present the measure of dependence, Kendall's tau and also provide the relationships between the tau and copulas [3].

\subsection{Copula and Conditional Distribution}

Definition 2.1. A two-dimensional copula is a function $C:[0,1] \times[0,1] \longrightarrow[0,1]$ with the following property:

1. $C(u, 0)=0=C(0, v) \quad$ for every $u, v \in[0,1]$.

2. $C(u, 1)=u$ and $C(1, v)=v \quad$ for every $u, v \in[0,1]$.

3. For every $u_{1}, u_{2}, v_{1}, v_{2} \in[0,1]$ with $u_{1} \leq u_{2}$ and $v_{1} \leq v_{2}$ :

$$
C\left(u_{2}, v_{2}\right)-C\left(u_{2}, v_{1}\right)-C\left(u_{1}, v_{2}\right)+C\left(u_{1}, v_{1}\right) \geq 0 .
$$

As such, it can present the joint distribution function of two standard uniform random variables $U_{1}, U_{2}$ :

$$
C\left(u_{1}, u_{2}\right)=\operatorname{Pr}\left(U_{1} \leq u_{1}, U_{2} \leq u_{2}\right)
$$

We can use this feature in order to re-write via copulas the joint distribution function of two (even non-uniform) random variables. The most interesting fact about copulas in this sense is the Sklar's theorem [9].

Theorem 2.1 (Sklar's Theorem). Let $H(x, y)$ be a joint distribution function with continuous marginals $F_{1}$ and $F_{2}$. Then there exists a copula $C$ with

$$
H(x, y)=C\left(F_{1}(x), F_{2}(y)\right)
$$

for every $x, y \in \mathbb{R}$. If $F_{1}$ and $F_{2}$ are continuous, then $C$ is unique. Otherwise, $C$ is uniquely determined on Range $F_{1} \times$ Range $F_{2}$. On the other hand, if $C$ is a copula and $F_{1}$ and $F_{2}$ are distribution functions, then the function $H(x, y)=$ $C\left(F_{1}(x), F_{2}(y)\right)$ is a joint distribution function with margins $F_{1}$ and $F_{2}$.

The theorem is very important, because it provides a way to analyze the dependence structure of bivariate distributions without studying marginal distributions. Copulas are thus bivariate uniform distributions, which describe the dependence 
structure of random variables. Indeed, for many financial applications, the problem is not to use a given bivariate distribution but consists in finding a convenient distribution to describe some stylized facts, for example the relationships between different asset returns. Beside as the literature mentioned, strictly increasing transformation of the underlying random variables result in the transformed variables having the same copula, which is very important for practical applications.

Theorem 2.2. Consider a copula $C(u, v)$, then

$$
\max (u+v-1,0) \leq C(u, v) \leq \min (u, v) .
$$

As a consequence of Sklar' theorem, if $X$ and $Y$ are random variables with a joint distribution function $H$ and margins $F$ and $G$, respectively, then for all $x, y \in R$, we have

$$
\max (F(x)+G(y)-1,0) \leq H(x, y) \leq \min (F(x), G(y))
$$

The above bounds are called the Fréchet-Hoeffding bounds for joint distribution functions $H$ with margins $F$ and $G$.

As we have known, dependency is an important concept inferring outcomes from a random variable based on the knowledge of a related factor. For an illustration, consider two uniform random variables $U$ and $V$ with a known copula $C$ and $U$ is observed. The goal is to deduce the conditional distribution which can then be used for predicting or estimating $V$. Assuming sufficient regularity, we obtain for the conditional cumulative distribution function

$$
\begin{aligned}
\operatorname{Pr}(V \leq v \mid U \leq u) & =\lim _{\delta \rightarrow 0} \frac{\operatorname{Pr}(V \leq v, U \in[u-\delta, u+\delta])}{\operatorname{Pr}(U \in[u-\delta, u+\delta])} \\
& =\lim _{\delta \rightarrow 0} \frac{C(u+\delta, v)-C(u-\delta, v)}{2 \delta} \\
& =\frac{\partial}{\partial u} C(u, v)
\end{aligned}
$$

Hence, the conditional cumulative distribution function is just the partial derivative function of copula and can be derived directly from the copula itself. The next theorem will give an explanation to the existence of the partial derivative of copulas.

Theorem 2.3. Let $C$ be a copula. For any $v \in[0,1]$, the partial derivative $\partial C / \partial u$ exists for almost all $u$, and for such $v$ and $u$,

$$
0 \leq \frac{\partial}{\partial u} C(u, v) \leq 1
$$


Similarly, for any $u \in[0,1]$, the partial derivative $\partial C / \partial v$ exists for almost all $v$, and for such $v$ and $u$,

$$
0 \leq \frac{\partial}{\partial v} C(u, v) \leq 1
$$

Furthermore, the functions $u \mapsto \partial C(u, v) / \partial u$ and are $v \mapsto \partial C(u, v) / \partial v$ defined and nondecreasing almost everywhere on $[0,1]$.

2.2. Some important Copulas It is time to present a few examples of copulas, especially for a particular class of copulas, the so-called Archimedean, which is particularly easy to handle and will be used in this paper.

2.2.1. Perfect dependence and independence The following three specific copulas are worth mentioning: the product copula, the minimum copula and the maximum copula. First, the product copula

$$
C(u, v)=u \cdot v
$$

related to the case of no dependence. As a direct consequence of Sklar's theorem we obtain that random variables are independent if and only if their copula is the product copula. As already mentioned, the Frechet-Hoeffding bounds are also related to copulas, where the maximum copula is the Frechet-Hoeffding upper bound which is given by

$$
C(u, v)=\min (u, v)
$$

Maximum copula refers to the case of perfect positive dependence. The other extreme is given by the minimum copula and as already mentioned, which is the Frechet-Hoeffding lower bound

$$
C(u, v)=\max (u+v-1,0)
$$

This case refers to perfect negative dependence.

2.2.2. Archimedean copulas Archimedean copulas may be constructed using a generating function $\phi:[0,1] \rightarrow[0, \infty]$ continuous, strictly decreasing, convex and such that $\phi(1)=0$. Given such a function $\phi$ a copula may be generated computing

$$
C(u, v)=\phi^{[-1]}(\phi(u)+\phi(v))
$$

where $\phi^{[-1]}$ is the pseudo-inverse of $\phi$, defined as

$$
\phi^{[-1]}(u)= \begin{cases}\phi^{-1}(u) & 0 \leq u \leq \phi(0) \\ 0 & \phi(0) \leq u \leq \infty\end{cases}
$$


Among Archimedean copulas, we are going to consider only the one-parameter ones, which are constructed using a generator $\phi_{\theta}(t)$, indexed by the parameter $\theta$. Now three kinds of Archimedean copulas will be introduced as follows.

Gumbel Copula The bivariate Gumbel copula or Gumbel-Hougaard copula is given by the function

$$
C_{\theta}^{G u}(u, v)=\exp \left(-\left[(-\ln u)^{\theta}+(-\ln v)^{\theta}\right]^{1 / \theta}\right)
$$

The parameter $\theta$ may take all values in the interval $[1, \infty)$. For $\theta=1$ we discover the product copula, while for $\theta \rightarrow \infty$ the Gumbel copula tends to the maximum copula so that the Gumbel copula interpolates between independence and perfect positive dependence.

Clayton Copula A second example is the Clayton copula, given by the function

$$
C_{\theta}^{C l}(u, v)=\left(u^{-\theta}+v^{-\theta}-1\right)^{-1 / \theta}
$$

where $\theta \in[-1,0) \cup(0, \infty)$. For the limits as $\theta \rightarrow 0$, we obtain the product copula, while for $\theta \rightarrow \infty$, the Clayton copula arrives as the maximum copula. For $\theta=$ -1 , we obtain the minimum copula. Thus, like the Gumbel copula, the Clayton copula interpolates between certain dependence structures, namely perfect negative dependence, independence and perfect positive dependence.

Frank Copula Another example is the Frank copula, given by

$$
C_{\theta}^{F r}(u, v)=-\frac{1}{\theta} \ln \left(1+\frac{\left(e^{-\theta u}-1\right)\left(e^{-\theta v}-1\right)}{e^{-\theta}-1}\right)
$$

where $\theta \in(-\infty, 0) \bigcup(0, \infty)$. For $\theta \rightarrow 0$ Frank copula reduces to the product copula, while it can reach the maximum copula and the minimum copula for $\theta \rightarrow \infty$ and $\theta \rightarrow-\infty$,respectively. Therefore, same with the Clayton copula, the Frank copula also interpolates between certain dependence structures, namely perfect negative dependence, independence and perfect positive dependence.

2.3. Measure of Concordance While a copula function fully describes the dependence structure between two random variables, we are often interested in simple summary statistics that indicate the extent and the direction of co-movement. The most basics of these measures are concordance measures, which assume the value of -1 for perfect negative dependence (corresponding to the lower Fréchet bound or the minimum copula), 0 for independence (corresponding to product copula) and 1 for perfect positive dependence (corresponding to the upper Fréchet bound or the 
maximum copula). Roughly speaking, two random variables are said to be concordant if large values of one come with large values of the other and small values of one come with small values of the other. Two samples $\left(x_{i}, y_{i}\right)$ and $\left(x_{j}, y_{j}\right)$ of a continuous random vector $(X, Y)$ are concordant if $\left(x_{i}-x_{j}\right)\left(y_{i}-y_{j}\right) \geq 0$. This means that either $x_{i} \leq x_{j}$ and $y_{i} \leq y_{j}$ or $x_{i} \geq x_{j}$ and $y_{i} \geq y_{j}$. Concordance measures are completely determined by the underlying copula and independent of the marginal distributions unlike Pearson's Correlation.

The two most common concordance measures are Kendall's tau and Spearman's rho. For two pairs of i.i.d. random vectors $\left(X_{1}, Y_{1}\right)$ and $\left(X_{2}, Y_{2}\right)$, Kendall's tau has an interpretation as the difference between the probability of a joint outcome with the same sign $\operatorname{Pr}\left[\left(\left(X_{1}-X_{2}\right)\left(Y_{1}-Y_{2}\right)>0\right)\right]$ and the probability of a joint outcome with different sign $\operatorname{Pr}\left[\left(\left(X_{1}-X_{2}\right)\left(Y_{1}-Y_{2}\right)<0\right)\right]$, in other words the difference between the probability of the variables either rising or falling together (concordance) minus the probability of them moving in different directions (discordance). Similarly, Spearman's rho measures the correlation between rank ordered data. The standard Pearson correlation coefficient is not a concordance measure and in particular, in contrast to Kendall's tau and Spearman's rho, it will be changed but non-monotonic transformations of the data since it is not independent of the marginal distributions.

Both measures have a lot of properties have a lot of properties in common. Being obviously measures with values in $[-1,1]$, they take the value 0 for independent variables (while there might also be non-independent random variables with zero rank correlation) and return $1(-1)$ for the perfect positive dependence (perfect negative dependence) case. Moreover, they can directly be derived from the unique copula $C$ that describes the dependence between two random variables $X$ and $Y$.

$$
\tau=4 \int_{0}^{1} \int_{0}^{1} C(u, v) d C(u, v)-1
$$

$$
\rho=12 \int_{0}^{1} \int_{0}^{1} C(u, v) d u d v-3
$$

In the following, the expression of $\tau$ as a function of the parameter $\theta$ of the copula are given in the following table, 


\begin{tabular}{|c|c|c|}
\hline Copula & $C(u, v ; \theta)$ & $\tau$ \\
\hline Gumbel & $\exp \left(-\left[(-\ln u)^{\theta}+\left(-\ln (v)^{\theta}\right]^{1 / \theta}\right)\right.$ & $\frac{\theta-1}{\theta}$ \\
\hline Clayton & $\left(u^{-\theta}+v^{-\theta}-1\right)^{-1 / \theta}$ & $\frac{\theta}{\theta+2}$ \\
\hline Frank & $-\frac{1}{\theta} \ln \left(1+\frac{\left(e^{-\theta u}-1\right)\left(e^{-\theta v}-1\right)}{e^{-\theta}-1}\right)$ & $1-\frac{4}{\theta}\left[1-D_{1}(\theta)\right]$ \\
\hline
\end{tabular}

where $D_{n}(x)=\frac{n}{x^{n}} \int_{0}^{x} \frac{t^{n}}{e^{t}-1} d t$ and $n$ is an integer.

\section{Forward and Futures Contracts}

Forward contract and futures contract are fundamentally similar. Both contracts are agreements between two parties to trade a specific good or asset at a future date at a strike price determined today. These agreements facilitate the transfer of risk between contracting parties, permitting the creation of hedging strategies [12].

In spite of their conceptual similarity, the implementation of these two contracts is quite different. Participants in futures markets trade highly standardized contracts by open outcry on organized exchanges. Clearing associations for the exchanges control default risk by assuming the opposite side of each transaction, guaranteeing payment. To reduce traders' incentive to default, the exchanges mark positions to market by settling contract gains and losses daily.

In contrast to the futures market, the market for forward contracts is mostly over the counter market. In the absence of a clearinghouse, trader reputation helps control default risk. Unlike futures contracts, no marking-to-market occurs: the parties in the contract make no payments until maturity of the contract. The forward market for financial interest rate instruments departs even further from the futures market format: a trader can use the cash market for interest rate instruments to establish a forward position. For example, a trader can secure a forward position for a three-month T-bill beginning three months in the future by buying a T-bill with a six-month maturity and selling a T-bill with a three-month maturity.

Some notions for forward contract and futures contract are given in the following. $T$ : maturity date of the forward and futures contract, $S(t)$ : price at time $\mathrm{t}$ of the good or asset on which the contracts are written, $R(t)$ : the interest rate at time $\mathrm{t}$, $B(t, T)$ : the price at time t of a zero-coupon bond paying 1 at time $\mathrm{T}$, $\operatorname{For}_{S}(t, T)$ : forward price at time $\mathrm{t}$ with maturity date $\mathrm{T}$, 
$F_{\text {ut }}(t, T)$ : futures price at time $\mathrm{t}$ with maturity date $\mathrm{T}$.

3.1. Forward Contract As already mentioned, forward contract is an agreement to buy or sell an asset at a certain future time for a certain price $K$. It is traded in the over-the counter market, usually between two financial institutions or between a financial institution and one of its clients. One of the parties to a forward contract assumes a long position and agrees to buy the underlying asset on a certain specified future date for a certain specified price. The other party assumes a short position and agrees to sell the asset on the same date for the same price.

Now, we are going to give the price formula of forward contract. First we define the discount process $D(t)=\exp \left(-\int_{0}^{t} R(u) d u\right)$, then the price at time $t$ of a zero-coupon bond paying 1 at time $T$ can be written as

$$
B(t, T)=\frac{1}{D(t)} \widetilde{\mathbb{E}}[D(T) \mid \mathcal{F}(t)]
$$

where $\widetilde{\mathbb{E}}$ is the risk-neutral expectation.

From the fact that the discounted asset price is a martingale under the risk-neutral measure $\widetilde{\mathbb{P}}$,

$$
\begin{aligned}
& \frac{1}{D(t)} \widetilde{\mathbb{E}}[D(T)(S(T)-K) \mid \mathcal{F}(t)] \\
& =\frac{1}{D(t)} \widetilde{\mathbb{E}}[D(T) S(T) \mid \mathcal{F}(t)]-\frac{K}{D(t)} \widetilde{\mathbb{E}}[D(T) \mid \mathcal{F}(t)] \\
& =S(t)-K B(t, T)
\end{aligned}
$$

This value should be zero and we have the following theorem.

Theorem 3.1. Assume that zero-coupon bonds of all maturities can be traded. Then the price of forward contract can be written as

$$
\text { For }_{S}(t, T)=\frac{S(t)}{B(t, T)}, \quad 0 \leq t \leq T .
$$

3.2. Futures Contract Like a forward contract, a future contract is an agreement between two parties to buy or sell an asset at a certain time in the future for a certain price. Unlike forward contracts, futures contracts are normally traded on an exchange. To make trading possible, the exchange specifies certain standardized features of the contract. As the two parties to the contract do not necessarily know each other, the exchange also provides a mechanism that gives the two parties a guarantee that the contract will be honored. Hence the futures price of an asset should be an adapted stochastic process $F u t_{S}(t, T)$ with two properties. 
(1) The futures price agrees with the asset price on the delivery date i.e.

$$
\operatorname{Fut}_{S}(T, T)=S(T) \text {. }
$$

(2) The value of holding the futures contract over a period of time and receiving the cash flows associated with this position is zero:

$$
\frac{1}{D\left(t_{0}\right)} \widetilde{\mathbb{E}}\left[\int_{t_{0}}^{t_{1}} D(u) d F u t_{S}(u, T) \mid \mathcal{F}(t)\right]=0, \quad 0 \leq t_{0} \leq t_{1} \leq T .
$$

The unique process satisfying these two properties can be defined as follows.

Definition 3.1. The futures price of an asset whose value at time $T$ is $S(t)$ is given by the formula

$$
\text { Fut }_{S}(t, T)=\widetilde{\mathbb{E}}[S(T) \mid \mathcal{F}(t)], \quad 0 \leq t \leq T .
$$

3.3. Forward-Futures Spread If the interest is a constant $r$, then $B(t, T)=$ $e^{-r(T-t)}$ and

$$
\begin{gathered}
\operatorname{For}_{S}(t, T)=\frac{S(t)}{B(t, T)}=e^{r(T-t)} S(t), \\
F u t_{S}(t, T)=e^{r T \widetilde{\mathbb{E}}}\left[e^{-r T} S(T) \mid \mathcal{F}(t)\right]=e^{r T} e^{-r t} S(t)=e^{r(T-t)} S(t) .
\end{gathered}
$$

In this case, the forward and futures prices agree.

When interest rates vary unpredictably (as they do in the real world), forward and futures prices are in theory no longer the same. We compare $\operatorname{For}_{S}(t, T)$ and $F u t_{S}(t, T)$ with a random interest rate. In this case, from the fact that

$$
B(t, T)=\widetilde{\mathbb{E}}[D(T) \mid \mathcal{F}(t)]
$$

we have $B(0, T)=\widetilde{\mathbb{E}}[D(T)]$, and the forward-futures spread can be written as

$$
\begin{aligned}
\text { For } \left._{S}(t, T)-\text { Fut }_{S}(t, T)\right) & =\frac{S(0)}{B(0, T)}-\widetilde{\mathbb{E}}[S(T)] \\
& =\frac{1}{\widetilde{\mathbb{E}}[D(T)]}(\widetilde{\mathbb{E}}[D(T) S(T)]-\widetilde{\mathbb{E}}[D(T)] \widetilde{\mathbb{E}}[S(T)]) \\
& =\frac{1}{B(0, T)} \widetilde{\operatorname{Cov}}[D(T), S(T)]
\end{aligned}
$$

where $\widetilde{\operatorname{Cov}}[D(T), S(T)]$ denotes the covariance of $D(T)$ and $S(T)$ under the riskneutral measure. If the interest rate is nonrandom, this covariance is zero and the futures price agrees with the forward price. 
Theorem 3.2. Under the risk-neutral measure with random interest rate, the price of forward-futures spread can be written as

$$
\left.F_{S o r}(t, T)-F u t_{S}(t, T)\right)=\frac{1}{B(0, T)} \widetilde{\operatorname{Cov}}[D(T), S(T)] \quad 0 \leq t \leq T .
$$

\section{Time Series Model Based on Copula}

Let $\left\{X_{t}\right\}$ be a stationary Markov process of order one. Then statistical properties are completely determined by the joint distribution of $X_{t-1}, X_{t}$, and $H\left(X_{t-1}, X_{t}\right)$. By Sklar's theorem, one can express $H\left(X_{t-1}, X_{t}\right)$ in terms of the marginal distribution of $X$ and the copula function of $X_{t-1}$ and $X_{t}$. As a result, the statistical properties of a stationary Markov process $\left\{X_{t}\right\}$ are completely determined by its marginal distribution and the copula of $X_{t-1}$ and $X_{t}$. This suggests the copula approach as an alternative approach to modeling a stationary Markov process: instead of specifying the joint distribution of $X_{t-1}$ and $X_{t}$, one specifies the marginal distribution of $X_{t}$ and the copula function of $X_{t-1}$ and $X_{t}$. The advantage of copula approach is that one has the freedom to choose the marginal distribution and the copula function separately; the former characterizes the marginal behavior such as the fat-tailed of the time series $\left\{X_{t}\right\}$, while the latter characterizes the temporal dependence property such as nonlinear, asymmetric dependence, of the time series.

Let $\left\{X_{t}\right\}_{t=1}^{n}$ be a sample from a stationary first order Markov process and let $F$ be the true invariant distribution, Suppose that $F$ is absolutely continuous. Let $C_{\theta}$ be the true parametric copula associated to the pair $\left(X_{t-1}, X_{t}\right)$ up to an unknown parameter $\theta$. In this setting, if one writes $U_{t}=F\left(X_{t}\right)$ and consider $\left\{U_{t}\right\}_{t=1}^{n}$, the transformed process is still a stationary Markov process, the joint distribution of $\left(U_{t-1}, U_{t}\right)$ will be $C_{\theta}\left(u_{t-1}, u_{t}\right)$ which can be written as according to Sklar's theorem:

$$
H\left(x_{t-1}, x_{t}\right)=C_{\theta}\left(F\left(x_{t-1}\right), F\left(x_{t}\right)\right)=C_{\theta}\left(u_{t-1}, u_{t}\right)
$$

As already mentioned, the conditional distribution of $X_{t}$ also can be written as

$$
H^{\prime}\left(x_{t} \mid x_{t-1}\right)=\operatorname{Pr}\left(X_{t} \leq x_{t} \mid X_{t-1} \leq x_{t}\right)=C^{\prime}\left(u_{t} \mid u_{t-1}\right)=\frac{\partial C_{\theta}\left(u_{t-1}, u_{t}\right)}{\partial u_{t-1}}
$$

After getting the conditional distribution of $X_{t}$, one can generate the time-series by using inverse function

$$
\begin{gathered}
u_{t}=C^{\prime-1}\left(\xi_{t} \mid u_{t-1}\right) \\
x_{t}=F^{-1}\left(u_{t}\right)
\end{gathered}
$$


where $\xi_{t}$ is the random number which follows $\mathbf{U}(0,1)$

Now it is very easy to simulate a time series from a copula-based Markov model. Let $c_{u_{t-1}}\left(u_{t} ; \theta\right)=\frac{\partial}{\partial u_{t-1}} C_{\theta}\left(u_{t-1}, u_{t}\right)$ be the conditional distribution of $U_{t}$ given $U_{t-1}=u_{t-1}$. To generate a series $\left\{X_{t}\right\}_{t=1}^{n}$ from a copula-based time series model, one may proceed as follows.

1. Choose the starting value $u_{0} \in[0,1]$.

2. Generate $n$ i.i.d. $\mathbf{U}(0,1)$ random variables $\left(r_{1}, r_{2}, \cdots, r_{n}\right)$.

3. For each $i=1,2, \cdots, n$, set $u_{i}=c_{u_{i-1}}^{-1}\left(r_{i} ; \theta\right)$.

4. For each $i=1,2, \cdots, n$, set $x_{i}=F^{-1}\left(u_{i}\right)$.

5 . The desired sample is $\left(x_{1}, x_{2}, \cdots, x_{n}\right)$.

The above algorithm can be used to generate a wide variety of dependence structures.

\section{Estimation of Forward-Futures Spread}

In this part, we evaluate marginal densities of the stock and stochastic interest rate to estimate the forward-futures spread between the forward price and the futures price with stochastic simulations.

5.1. Computation of all Statistics and Marginal Densities The data on which our investigation is based are the historical stock price (SP500) and interest rate (LIBOR) ranging from 2 Jan. 1986 until 29 Dec. 2006, totally 5298 observations. The following table presents the sample moments of these time series, and figure 1 displays these two time series. For convenience, we denote the stock price as $S(t)$ and the interest rate as $R(t)$.

\begin{tabular}{|c|c|c|c|c|}
\hline Time series & Min & Max & Mean & Std.dev \\
\hline$S(t)$ & 203.49 & 1527.50 & 755.05 & 407.27 \\
\hline$R(t)$ & 0.9900 & 11.3750 & 5.5058 & 2.1684 \\
\hline
\end{tabular}

Form the historical data we obtain the marginal density function of each time series reported in figure 2 and figure 3, respectively, and the following table explains the marginals for them.

\begin{tabular}{|c|c|c|}
\hline Time series & Estimated distribution & Parameter \\
\hline$S(t)$ & Lognormal & $\mu=6.4579$ and $\sigma=0.6034$ \\
\hline$R(t)$ & Normal & $\mu=5.5058$ and $\sigma=2.1684$ \\
\hline
\end{tabular}



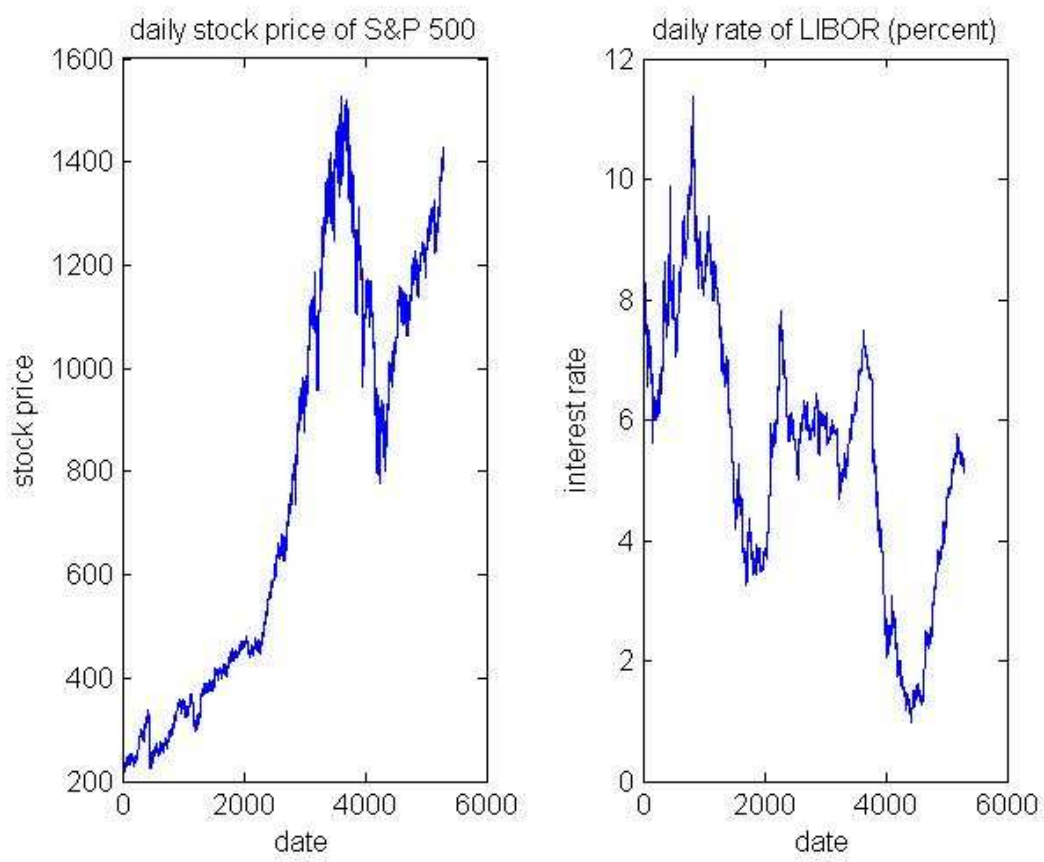

Figure 1. Scatter plots for marginal distribution

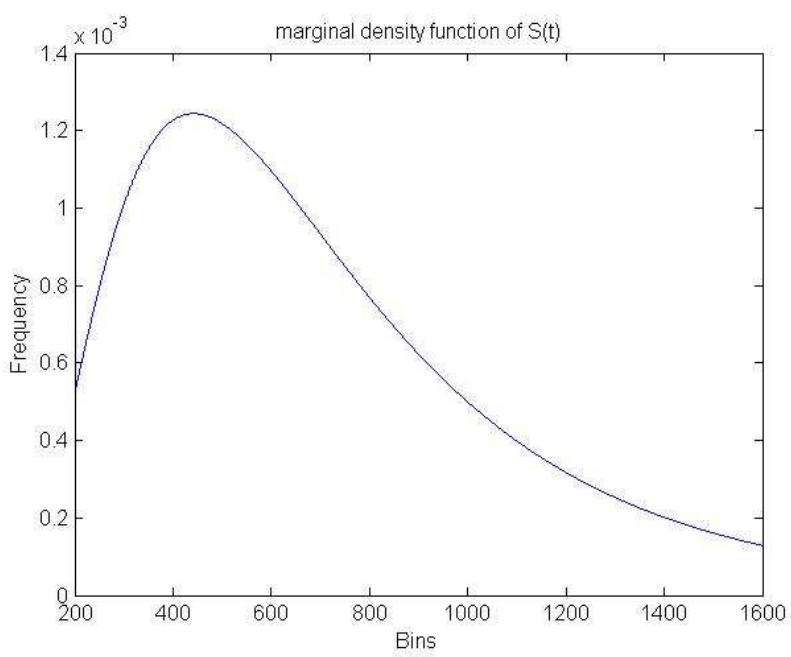

Figure 2. Marginal density for stock price

We have an intuitive impression of the dependence structure for each time series by graphical statistical analysis previously. And also we can have a further understand of such a dependence structure through calculating the concordance measure, 


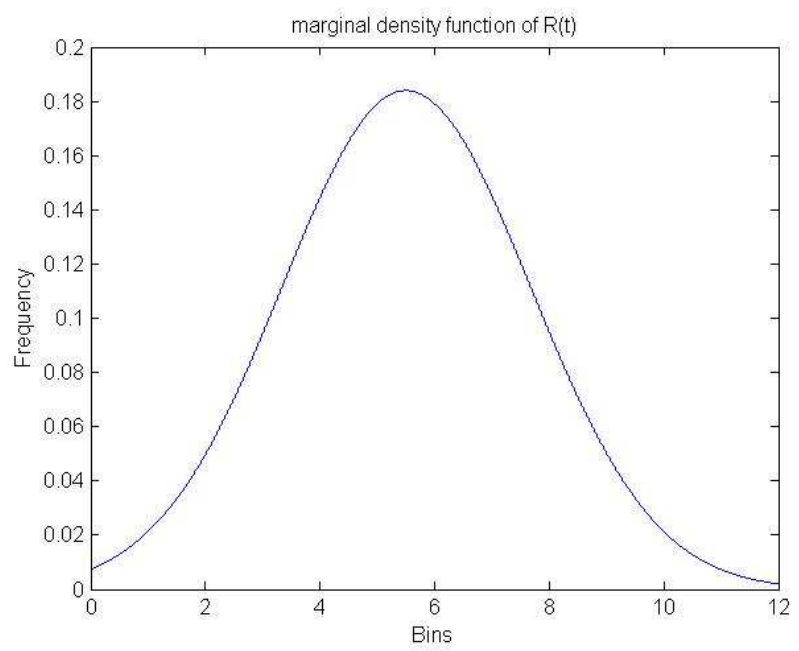

Figure 3. Marginal density for interest rate

Kendall's tau. However, recalling that these statistics are particularly case of copulas' application, the copula notion extends the concordance notion to the continuous framework. Moreover, even if the two concordance measures mentioned above allow to measure the association degree between two random variables, these statistics provide less information than a copula. In the following table, we report the estimated parameters $\theta$ for the families of Archimedean copulas described previously, as well as the measure concordance, Kendall's tau.

\begin{tabular}{|c|c|c|c|c|}
\hline Sample of pairs & Kendall's $\tau$ & Gumbel's $\theta$ & Clayton's $\theta$ & Frank's $\theta$ \\
\hline$\left[S\left(t_{j-1}\right), S\left(t_{j}\right)\right]$ & 0.9847 & 65.3595 & 128.7190 & 259.7825 \\
\hline$\left[R\left(t_{j-1}\right), R\left(t_{j}\right)\right]$ & 0.9843 & 63.6943 & 125.3885 & 253.1214 \\
\hline
\end{tabular}

5.2. Computation of Prices of Spread In what follows we will use Gumbel copula for simulating the time series. Here again, we write the form of Gumbel copula, and discover its partial derivative function.

$$
C_{\theta}^{G u}(u, v)=\exp \left(-\left[(-\ln u)^{\theta}+(-\ln v)^{\theta}\right]^{1 / \theta}\right)
$$

$\left.\frac{\partial}{\partial u} C_{\theta}^{G u}(u, v)=\exp \left(-\left[(-\ln u)^{\theta}+(-\ln v)^{\theta}\right]^{1 / \theta}\right)\left[(-\ln u)^{\theta}+(-\ln v)^{\theta}\right]^{(1-\theta) / \theta}\right) \frac{(-\ln u)^{\theta-1}}{u}$

We simulate 2000 times for $S(t)$ and $R(t)$ based on the above algorithm, then we get 2000 paths for each process. We take average after calculating the covariance of 
$S(t)$ and $D(t)$ and the bond price $B(0, t)$.

$$
\begin{gathered}
\widetilde{\operatorname{Cov}}[D(T), S(T)]=\frac{1}{n} \sum_{i=1}^{n} \widetilde{\operatorname{Cov}}\left[D_{i}(t), S_{i}(t)\right] \\
B(0, T)=\frac{1}{n} \sum_{i=1}^{n} B_{i}(0, T)
\end{gathered}
$$

where $D_{i}(t), S_{i}(t)$ and $B_{i}(0, T)$ is the value of $D(T), S(T)$ and $B(0, T)$ for the $i$ th path.

We vary $T$ between 0.25 and 10, i.e. between three months and ten years, the values of the forward-futures spread are shown in the following table and figure 4 .

\begin{tabular}{|c|c|c|c|c|c|c|c|}
\hline $\mathrm{T}$ & 0.25 & 0.5 & 1 & 2 & 3 & 5 & 10 \\
\hline$\widetilde{\operatorname{Cov}[D(T), S(T)]}$ & 0.0079 & 0.0399 & 0.1248 & 0.3593 & 0.8310 & 2.3450 & 8.3884 \\
\hline$B(0, T)$ & 0.9871 & 0.9745 & 0.9498 & 0.9028 & 0.8586 & 0.7771 & 0.6071 \\
\hline Spread & 0.0080 & 0.0409 & 0.1314 & 0.3980 & 0.9679 & 3.0176 & 13.8172 \\
\hline
\end{tabular}

It is noticeable that the price of the spread is very small, almost negligible, for time horizons up to one year. However, it grows exponentially thereafter. For a 10-year time horizon, the price of the spread is about 100 times bigger than that of the 1-year time horizon. This means that the forward-futures spread should be taken into account for contracts with long maturities.

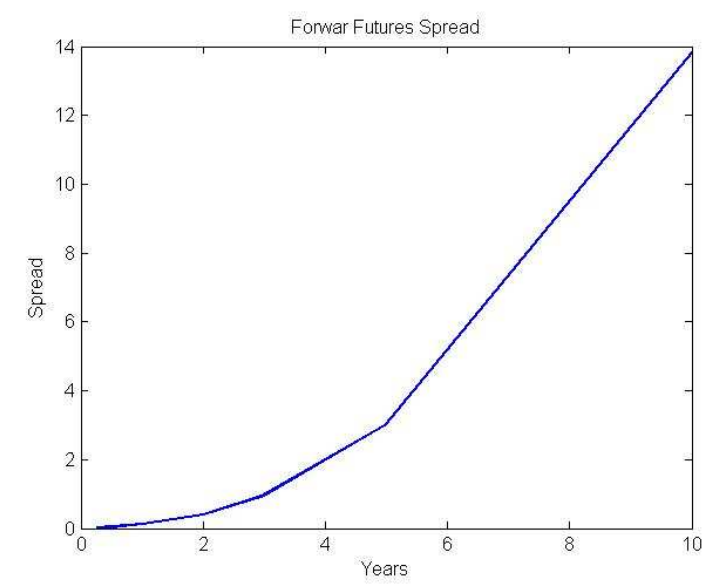

Figure 4. The price of forward-futures spread 


\section{Conclusion}

In this paper, our main goal is to estimate the spread between the price of a forward contract and the price of a similar futures contract. We have studied temporal dependence properties of a class of stationary Markov time series: a member of this class is completely characterized by a parametric copula and a nonparametric marginal distribution. We investigated and used this algorithm to simulate two time series, stock price and interest rate, respectively, which have led us to studies of the pricing differences between forward and futures contracts. The theoretical differences between forward and futures prices for contracts that last only a few months even one year are in most circumstances sufficiently small to be ignored. In this case we can assume the forward price agrees with the futures price for convenience. However, as the life of a futures contract increases, the differences between forward and futures contracts are liable to become significant. It is then dangerous to assume that forward and futures prices are perfect substitutes for each other. Therefore, it is necessary to know the spread between them. We suggest the estimation of the price of spread of forward and futures contracts by using copula functions even if the forward contract can hardly be shown in financial markets.

\section{REFERENCES}

1. Chen, X. \& Fan, Y.: Estimation of Copula-Based Semi-parametric Time Series Model. Journal of Econometrics 130 (2006), 307-335.

2. Cox, J.C.; Ingersoll Jr, J.E. \& Ross, S.A.: The relation between forward prices and futures prices. Journal of Financial Economics. 9 (1981), no. 4, 321-346

3. Fredricks, G.A. \& Nelsen, R.B.: On the relationship between Spearman's rho and Kendall's tau for pairs of continuous random variables. Journal of Statistical Planning and Inference 137 (2007), no. 7, 2143-2150.

4. Genest, C. \& Rivest, L.P.: Statistical inference procedures for bivariate Archimedean copulas. Journal of the American Statistical Association 88 (1993), no. 423, 1034-1043.

5. Grinblatt, M. \& Jegadeesh, N.: Relative pricing of Eurodollar futures and forward contracts. The Journal of Finance 51 (1996), no. 4, 1499-1522.

6. J.C. Hull: Options, Futures and Other Derivatives. Prentice Hall, 2008.

7. Kendall, M.G.: A new measure of rank correlation. Biometrika 30 (1938), no. 1-2, 81-93.

8. Murawski, C.: On the Forward-Futures Spread and Default Risk. Available at SSRN 389281,2003 
9. Nelsen, R.B.: An Introduction to Copulas. New York: Springer, 2nd edition, 2005.

10. Pumi, G. \& Lopes, S.R.: Simulation of Univariate Time Series Using Copulas. Preprint.

11. Schmidt, T.: Coping with copulas. Chapter forthcoming in Risk Books: Copulas-from theory to applications in finance, 2006.

12. Shreve, S.E.: Stochastic Calculus for Finance II - Continuous-Time Models. New York: Springer, 2004.

åDepartment of Mathematics, Sungkyunkwan University, Suwon, Gyeonggi 440-746, KOREA

Email address: magie609@gmail.com

${ }^{\text {b}}$ Department of Mathematics, Sungkyunkwan University, Suwon, Gyeonggi 440-746, Korea

Email address: skim@skku.edu 